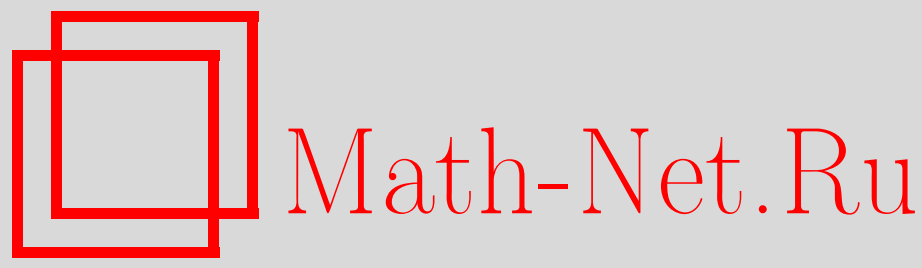

О. Литт, Y. Х. Бассичис, Модифицированный метод Боголюбова в применении к простой бозонной модели, ТМФ, 2017, том 190, номер 1, 124-137

DOI: https://doi.org/10.4213/tmf9088

Использование Общероссийского математического портала Math-Net.Ru подразумевает, что вы прочитали и согласны с пользовательским соглашением http: //www . mathnet.ru/rus/agreement

Параметры загрузки:

IP: 54.197 .130 .99

26 апреля 2023 г., $17: 38: 27$

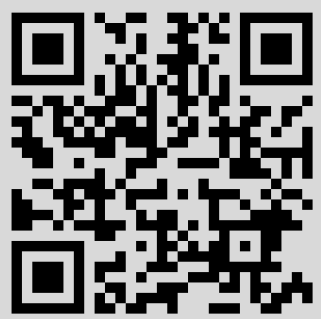




\title{
МОДИФИЦИРОВАННЫЙ МЕТОД БОГОЛЮБОВА В ПРИМЕНЕНИИ К ПРОСТОЙ БОЗОННОЙ МОДЕЛИ
}

\begin{abstract}
При помощи не являющейся калибровочно-инвариантной модификации точного гамильтониана получен аналог гамильтонианиана для простой точно решаемой бозонной модели. Собственные значения найденного оператора близки к собственным значениям исходного гамильтониана. Одночастичное приближение к новому двухчастичному оператору проводится в духе боголюбовской аппроксимации. Так как возникают только операторы числа частиц, не требуется искать отдельные $c$-численные аппроксимации для операторов рождения и уничтожения в основном состоянии. Для простой модели результаты новой аппроксимации по целому ряду параметров ближе к точным значениям, чем при применении стандартного метода Боголюбова. Превосходство в точности сильно возрастает при увеличении сил взаимодействия в модели.
\end{abstract}

Ключевые слова: модифицированная боголюбовская аппроксимация.

DOI: $10.4213 / \operatorname{tmf} 9088$

\section{1. ВВЕДЕНИЕ}

С экспериментальным открытием конденсации Бозе-Эйнштейна в разреженом газе [1] вновь возник интерес к теоретическому объяснению данного явления. Теоретические достижения в этой области хорошо освещены в обзоре Загребнова и Бру [2] и книгах [3] и [4]. Основополагающей для теоретического анализа являлась идея Боголюбова [5]. Так как из конденсации следует, что основное состояние системы бозонов было макроскопически занятым, операторы рождения и уничтожения для этого состояния можно приблизить $c$-числами. Более того, входящими в гамильтониан слагаемыми, не содержащими операторы основного состояния, можно пренебречь. Точность этих приближений интенсивно изучалась в различных предельных случаях [2]. Для изучения слабо взаимодействующих захваченных бозонов были построены обобщения теории Боголюбова [6]. Десятилетия назад Фолди и Бассичис [7] предложили модель для частиц Бозе-Эйнштейна, ограниченных размерностью единица, где существуют только одно основное состояние и одно возбужденное.

*Texas A\&M University, College Station, Texas, United States of America. E-mail: oplitt@yahoo.com, bassichis_w@hotmail.com 
Гамильтониан модели оказался точно решаемым и потому был применен для проверки боголюбовских аппроксимаций.

Простая двухуровневая модель с тех пор использовалась для многих целей, в частности для проверки различных других аппроксимационных схем [8]. В последнее время возник интерес к одномерным бозонным моделям из-за физической реализуемости систем, в которых бозоны, в частности ${ }^{4} \mathrm{He}$, ограничены в квазиодномерной среде [9]. В оригинальной работе предлагаемое улучшение боголюбовской аппроксимации, основанное на казалось бы более точной численной аппроксимации, при проверке неожиданно показало бо́льшее расхождение с точными значениями, чем боголюбовская аппроксимация. Так как предлагавшееся улучшение основывалось лишь на численном приближении, оно не имело бы большого значения для более общих проблем, связанных с бозонами, даже если бы оно и показало лучшие результаты.

В настоящей работе мы предлагаем другую схему аппроксимации, основанную на анзаце и, возможно, имеющую более широкую область применения. Мы начинаем с подходящей для этого случая процедуры, переводящей с помощью не являющегося калибровочно-инвариантным преобразования гамильтониан в другой оператор. После этого полученный двухчастичный оператор можно приблизить одночастичным оператором аналогично тому, как это делается в рамках боголюбовской аппроксимации, в которой операторы основного и возбужденного состояний рассматриваются по отдельности. Показано, что такая аппроксимация приводит к более точным результатам, чем результаты, получающиеся при боголюбовской аппроксимации, в широком диапазоне сил взаимодействия и числа частиц. Возможно, эта аппроксимация окажется полезной и для более реалистичных моделей, в которых точные решения недостижимы и требуется применение аппроксимаций.

\section{2. ОДНОМЕРНАЯ БОЗОННАЯ МОДЕЛЬ}

Рассматриваемая система подробно описана в оригинальной статье. Имеются всего три состояния для частиц: основное с нулевой кинетической энергией и два возбужденных. Возбужденные состояния имеют равные, но противоположно направленные импульсы, кинетическая энергия единственной возбужденной частицы полагается равной единице. Операторы рождения и уничтожения для основного состояния обозначаются как $a_{0}^{\dagger}, a_{0}$, а для возбужденных состояний $-a_{+}^{\dagger}, a_{+}$и $a_{-}^{\dagger}, a_{-}$. В простейшем варианте модели предполагается, что возбужденные частицы не взаимодействуют. В этом случае гамильтониан имеет вид

$$
H=a_{+}^{\dagger} a_{+}+a_{-}^{\dagger} a_{-}+g\left[a_{0}^{\dagger} a_{0}\left(a_{+}^{\dagger} a_{+}+a_{-}^{\dagger} a_{-}\right)+\left(a_{0}^{\dagger}\right)^{2} a_{+} a_{-}+a_{0}^{2} a_{+}^{\dagger} a_{-}^{\dagger}\right] .
$$

Существуют два оператора, коммутирующих с гамильтонианом: операторы числа частиц $N_{\text {ор }}=a_{0}^{\dagger} a_{0}+a_{+}^{\dagger} a_{+}+a_{-}^{\dagger} a_{-}$и $\Delta_{\text {ор }}=a_{+}^{\dagger} a_{+}-a_{-}^{\dagger} a_{-}$. В оригинальной статье гамильтониан действовал на пространстве, заданном собственными значениями операторов $N_{\text {ор }}, \Delta_{\text {ор }}$ и $a_{-}^{\dagger} a_{-}$, обозначенном $\left|N, \Delta, n_{-}\right\rangle$. Для простоты будем рассматривать только состояния с $\Delta=0$. Для системы $N$ частиц результирующую квадратную матрицу размера $(N / 2+1) \times(N / 2+1)$ можно диагонализовать для получения точных энергий и волновой функции основного состояния, а также всего 
спектра. Для наших целей удобнее записывать базисные состояния без нормирующего множителя:

$$
\left|n_{0}, n_{+}, n_{-}\right\rangle=\left(a_{0}^{\dagger}\right)^{n_{0}}\left(a_{+}^{\dagger}\right)^{n_{+}}\left(a_{-}^{\dagger}\right)^{n_{-}}|0\rangle,
$$

и для специального случая $\Delta=0$ базис принимает вид

$$
\left|n_{0}, n\right\rangle=\left(a_{0}^{\dagger}\right)^{n_{0}}\left(a_{+}^{\dagger}\right)^{n}\left(a_{-}^{\dagger}\right)^{n}|0\rangle .
$$

В базисе (3) матричные элементы гамильтониана не равны нулю только для состояний с одинаковым полным числом частиц, т. е. при $n_{0}^{\prime}+2 n^{\prime}=n_{0}+2 n$. Логично пронумеровать базисные состояния для построения матрицы в соответствии с числом $N=n_{0}+2 n$. Тогда бесконечная матрица состоит из блоков, стоящих на диагонали, размерности $(N / 2+1) \times(N / 2+1), 0<N<\infty$.

\section{3. БОГОЛЮБОВСКАЯ АППРОКСИМАЦИЯ}

Рассматриваемую модель можно использовать для проверки боголюбовской аппроксимации, показавшей свою полезность для широкого ряда приложений [3]. В рамках этой аппроксимации все операторы в основном состоянии заменяются с-числами:

$$
a_{0}^{\dagger} \rightarrow N_{0}^{1 / 2}, \quad a_{0} \rightarrow N_{0}^{1 / 2}
$$

Получающийся одночастичный оператор $h_{\mathrm{B}}$, где строчная буква в его обозначении указывает на то, что это одночастичный оператор, имеет вид

$$
h_{\mathrm{B}}=a_{+}^{\dagger} a_{+}+a_{-}^{\dagger} a_{-}+g N_{0}\left[a_{+}^{\dagger} a_{+}+a_{-}^{\dagger} a_{-}+a_{+} a_{-}+a_{+}^{\dagger} a_{-}^{\dagger}\right] .
$$

Оператор (5) можно диагонализовать с помощью канонического преобразования. Это даст значение энергии основного состояния, волновую функцию $|g s\rangle$ и точный спектр состояния. Оператор (5) больше не коммутирует с оператором полного числа частиц, так что число $N$ не является подходящей квантовой величиной. Параметр $N_{0}$ описывает число частиц в состоянии с нулевым импульсом. Его можно считать равным числу $N$, на которое не влияет истощение основного состояния. Более точную аппроксимацию можно получить, если потребовать, чтобы среднее полное число частиц системы, определенное как $N_{0}+\left\langle g s\left|a_{+}^{\dagger} a_{+}+a_{-}^{\dagger} a_{-}\right| g s\right\rangle$, равнялось желаемому полному числу частиц системы $N$. Это можно осуществить, используя итеративную процедуру.

По-другому боголюбовские результаты можно получить посредством диагонализации оператора $h_{\mathrm{B}}$ в подходящем базисе. Использование блока состояний на диагонали пространства, относящегося к точному гамильтониану, не приводит к удобному способу получения результата Боголюбова. Это следует из того, что $h_{\mathrm{B}}$ не сохраняет полного числа частиц. Более того, так как $h_{\mathrm{B}}$ не содержит операторов в состоянии нулевого импульса, для единственных ненулевых матричных элементов верно равенство $n_{0}^{\prime}=n_{0}$, а значит, слагаемые в блоках с фиксированным номером $N$ на диагоналях с номерами $\left\langle n_{0}, n^{\prime}\left|a_{+} a_{-}\right| n_{0}, n\right\rangle$ и $\left\langle n_{0}, n^{\prime}\left|a_{+}^{\dagger} a_{-}^{\dagger}\right| n_{0}, n\right\rangle$ равны нулю при

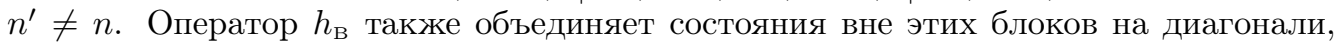
так что нумерация состояний, которая была удобной для точного гамильтониана, приведет к образованию матрицы бесконечного размера.

Если вместо этого перенумеровать базисные состояния так, чтобы все состояния с номером, равным $n_{0}$, были сгруппированы и затем упорядочены по возрастанию 
номера $n$, то вся матрица окажется состоящей из бесконечного числа несвязанных идентичных матриц, характеризующихся параметром $n_{0}$. Матрицы идентичны, поскольку все матричные элементы не зависят от $n_{0}$. Хотя нам нужно диагонализовать только одну такую матрицу, матрица не "обрезается" по числу рассматриваемых частиц, как это происходит для точного гамильтониана. То, что это ведет к возможному существованию компонент собственной функции, имеющих в возбужденном состоянии бо́льшее число частиц, чем имеется в системе, является разумным, только если не найдется положительного значения $N_{0}$, удовлетворяющего указанному выше уравнению для $N$. На практике, однако, не требуется диагонализовать бесконечную матрицу. Для системы $N$ частиц изменение минимального собственного значения матрицы при разложении на более чем $N / 2+1$ слагаемых можно найти посредством прямого вычисления, и, как показано ниже, оно является малым. Этот факт также можно понять исходя из теории возмущений, так как вклад минимального собственного значения, включающего состояние $|k\rangle$, обратно пропорционален величине $E_{0}-E_{k}$, быстро растущей при $k>N / 2+1$.

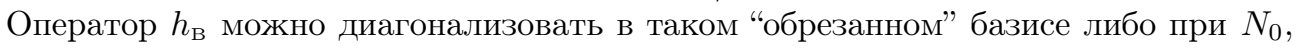
равном числу частиц, т. е. используя аппроксимацию без учета истощения, либо считая, что $N_{0}$ - параметр. Вместо определения $N_{0}$ с помощью итераций его можно получить посредством диагонализации матрицы для разных значений $N_{0}$, чтобы определить значение, отвечающее желаемому значению $N$, с помощью соотношения

$$
N=N_{0}+\left\langle a_{+}^{\dagger} a_{+}+a_{-}^{\dagger} a_{-}\right\rangle \text {. }
$$

Среднее здесь находится с использованием собственной функции основного состояния. Эта процедура дает результат, практически совпадающий с результатом канонического преобразования, если размер базиса положить равным $N / 2+1$, а малые поправки, возникающие при использовании базисов больших размеров, не влияют на приведенный результат.

\section{4. ДРУГОЙ ПОДХОД}

В попытке найти уточнение боголюбовской аппроксимации точный гамильтониан можно разделить на две части. Первая часть, $H_{0}$, сохраняет число частиц в основном состоянии, вторая, $H_{\mathrm{P}}$, изменяет число частиц в основном состоянии:

$$
\begin{aligned}
& H_{0}=a_{+}^{\dagger} a_{+}+a_{-}^{\dagger} a_{-}+g a_{0}^{\dagger} a_{0}\left(a_{+}^{\dagger} a_{+}+a_{-}^{\dagger} a_{-}\right), \\
& H_{\mathrm{P}}=g a_{0}^{\dagger} a_{+} a_{-}+g a_{0}^{2} a_{+}^{\dagger} a_{-}^{\dagger} .
\end{aligned}
$$

В качестве первого шага в рассматриваемой процедуре эти операторы можно представить в виде скалярных произведений в евклидовой метрике:

$$
\begin{aligned}
& H_{0}=\left(a_{+}^{\dagger}, a_{-}^{\dagger}, g a_{0}^{\dagger} a_{0}, g a_{0}^{\dagger} a_{0}\right)\left(\begin{array}{cccc}
1 & 0 & 0 & 0 \\
0 & 1 & 0 & 0 \\
0 & 0 & 1 & 0 \\
0 & 0 & 0 & 1
\end{array}\right)\left(\begin{array}{c}
a_{+} \\
a_{-} \\
a_{+}^{\dagger} a_{+} \\
a_{-}^{\dagger} a_{-}
\end{array}\right), \\
& H_{\mathrm{P}}=g\left(a_{0}, a_{0}^{\dagger}\right)\left(\begin{array}{ll}
1 & 0 \\
0 & 1
\end{array}\right)\left(\begin{array}{c}
a_{0} a_{+}^{\dagger} a_{-}^{\dagger} \\
a_{0}^{\dagger} a_{+} a_{-}
\end{array}\right) .
\end{aligned}
$$


Наша цель - получить одночастичный оператор боголюбовского типа, который может иметь приложения за рамками данной модели. Даже если чисто численное улучшение аппроксимации, предложенное в статье [7], приводит к поправкам в правильном направлении, оно не допускает дальнейшего обобщения. В этой связи была предложена следующая процедура. Вместо использования евклидовой метрики с оператором $H_{\mathrm{P}}$ предлагается рассмотреть оператор, получаемый при замене этой метрики на отражающую метрику $\left(\begin{array}{ll}0 & 1 \\ 1 & 0\end{array}\right)$. Соответствующий оператор имеет вид

$$
H_{\mathrm{R}}=g\left(a_{0}, a_{0}^{\dagger}\right)\left(\begin{array}{ll}
0 & 1 \\
1 & 0
\end{array}\right)\left(\begin{array}{c}
a_{0} a_{+}^{\dagger} a_{-}^{\dagger} \\
a_{0}^{\dagger} a_{+} a_{-}
\end{array}\right)
$$

Полный аналог гамильтониана $\widetilde{H}=H_{0}+H_{\mathrm{R}}$ при такой замене записывается как

$$
\widetilde{H}=a_{+}^{\dagger} a_{+}+a_{-}^{\dagger} a_{-}+g\left[a_{0}^{\dagger} a_{0}\left(a_{+}^{\dagger} a_{+}+a_{-}^{\dagger} a_{-}\right)+a_{0} a_{0}^{\dagger} a_{+} a_{-}+a_{0}^{\dagger} a_{0} a_{+}^{\dagger} a_{-}^{\dagger}\right]
$$

где символ $\widetilde{H}$ используется для указания на то, что этот оператор более не является гамильтонианом.

Так как целью является получение одночастичной аппроксимации двухчастичного оператора аналогично тому, как это сделано в боголюбовской аппроксимации, операторы рождения и уничтожения для основного состояния исключаются. Эти операторы возникают в операторе $\widetilde{H}$ только в комбинациях, при которых сохраняется число частиц, в отличие от ситуации, когда они появляются в боголюбовском гамильтониане. Боголюбовский способ нумерации базисных состояний подходит и для этого случая, так как операторы не связывают состояния с различными значениями $n_{0}$, но связывают состояния с разным числом частиц.

Для системы $N$ частиц оператор числа частиц $a_{0}^{\dagger} a_{0}$ предлагается заменить на операторы

$$
a_{0}^{\dagger} a_{0}=N-a_{+}^{\dagger} a_{+}-a_{-}^{\dagger} a_{-}
$$

и

$$
a_{0} a_{0}^{\dagger}=a_{0}^{\dagger} a_{0}+1=N+1-a_{+}^{\dagger} a_{+}-a_{-}^{\dagger} a_{-} .
$$

Здесь $N$ теперь - не параметр, аналогичный боголюбовскому $N_{0}$, а полное число частиц системы. Далее, так как $a_{0}^{\dagger} a_{0}$ есть оператор числа частиц и не может иметь отрицательных собственных значений, то же самое условие должно выполняться и для заменяющих операторов.

При данной замене аналогичный гамильтониану оператор можно записать как

$$
\begin{aligned}
\widetilde{H}= & a_{+}^{\dagger} a_{+}+a_{-}^{\dagger} a_{-}+g\left[\left(N-a_{+}^{\dagger} a_{+}-a_{-}^{\dagger} a_{-}\right)\left(a_{+}^{\dagger} a_{+}+a_{-}^{\dagger} a_{-}\right)\right]+ \\
& +g\left[\left(N+1-a_{+}^{\dagger} a_{+}-a_{-}^{\dagger} a_{-}\right) a_{+} a_{-}+\left(N-a_{+}^{\dagger} a_{+}-a_{-}^{\dagger} a_{-}\right) a_{+}^{\dagger} a_{-}^{\dagger}\right] .
\end{aligned}
$$




\section{5. РЕЗУЛЬТИРУЮЩИЕ МАТРИЦЫ}

Ненулевые элементы точного гамильтониана в пространстве $\left|n_{0}, n\right\rangle$ имеют вид

$$
\begin{aligned}
\left\langle n_{0}, n\left|H_{E}\right| n_{0}, n\right\rangle & =2 n+2 g n_{0} n, \\
\left\langle n_{0}+2, n-1\left|H_{E}\right| n_{0}, n\right\rangle & =g n\left(n_{0}+1\right)^{1 / 2}\left(n_{0}+2\right)^{1 / 2}, \\
\left\langle n_{0}-2, n+1\left|H_{E}\right| n_{0}, n\right\rangle & =g(n+1)\left(n_{0}-1\right)^{1 / 2} n_{0}^{1 / 2} .
\end{aligned}
$$

Боголюбовский аналог гамильтониана имеет ненулевые матричные элементы только между состояниями с одинаковыми значениями $n_{0}$, причем не зависящие от $n_{0}$. Ненулевые матричные элементы в пространстве $\left|n_{0}, n\right\rangle$ с фиксированным $n_{0}$ суть

$$
\begin{aligned}
\left\langle n\left|h_{\mathrm{B}}\right| n\right\rangle & =2 n+2 g N_{0} n, \\
\left\langle n-1\left|h_{\mathrm{B}}\right| n\right\rangle & =g n N_{0}, \\
\left\langle n+1\left|h_{\mathrm{B}}\right| n\right\rangle & =g(n+1) N_{0},
\end{aligned}
$$

где вновь $N_{0}$ - полное число частиц в системе, где истощение не учитывается. Иначе $N_{0}$ - это параметр, представляющий число частиц в основном состоянии, который определяется так, чтобы среднее числа частиц в системе совпадало с желаемым полным числом частиц. Аналог гамильтониана, полученный с использованием отражающей метрики $\left(\begin{array}{ll}0 & 1 \\ 1 & 0\end{array}\right)$, имеет ненулевые матричные элементы между теми же состояниями, что и боголюбовский оператор. Значение $n_{0}$ должно быть таким же, чтобы два состояния были связаны и матричные элементы не зависели от $n_{0}$. Они имеют вид

$$
\begin{aligned}
\langle n|\widetilde{H}| n\rangle & =2 n+2 g n(N-2 n), \\
\langle n-1|\widetilde{H}| n\rangle & =g n(N+3-2 n), \\
\langle n+1|\widetilde{H}| n\rangle & =g(n+1)(N-2-2 n),
\end{aligned}
$$

где $N$ - полное число частиц в системе.

При том, что в рамках боголюбовской аппроксимации предлагаются замены

$$
n_{0} \rightarrow N_{0}, \quad\left(n_{0}+1\right)^{1 / 2}\left(n_{0}+2\right)^{1 / 2} \rightarrow N_{0}, \quad\left(n_{0}-1\right)^{1 / 2} n_{0}^{1 / 2} \rightarrow N_{0},
$$

попытка ее улучшения, предложенная в статье [7], основывается на заменах

$$
\left(n_{0}+1\right)^{1 / 2}\left(n_{0}+2\right)^{1 / 2} \rightarrow N_{0}+2, \quad\left(n_{0}-1\right)^{1 / 2} n_{0}^{1 / 2} \rightarrow N_{0}, \quad n_{0} \rightarrow N_{0} .
$$

Использование этих замен привело к результатам, которые сильнее отличаются от точных значений, чем результаты, основанные на боголюбовской аппроксимации.

Матричные элементы оператора $\widetilde{H}$ отвечают заменам

$$
\left(n_{0}+1\right)^{1 / 2}\left(n_{0}+2\right)^{1 / 2} \rightarrow N-2 n+3, \quad\left(n_{0}-1\right)^{1 / 2} n_{0}^{1 / 2} \rightarrow N-2 n-2, \quad n_{0} \rightarrow N-2 n,
$$

близким по духу к заменам из статьи [7]. Получающиеся собственные значения, отвечающие энергиям основного и возбужденного состояний, близки к точным значениям. Хотя данная процедура дает аппроксимирующий оператор с собственными 
значениями, более близкими к точным значениям, чем при боголюбовской аппроксимации, она не дает выигрыша при проведении точных вычислений, так как оператор все еще является двухчастичным. Преимущество боголюбовской аппроксимации состоит в том, что в ней участвует одночастичный оператор, матричные значения которого можно найти с куда меньшими усилиями, чем это требуется для двухчастичных операторов. Одночастичное приближение для $\widetilde{H}$ получено в следующем разделе.

\section{6. АППРОКСИМАЦИЯ БОГОЛЮБОВСКОГО ТИПА}

В духе боголюбовской аппроксимации двухчастичный оператор $\widetilde{H}$ можно свести к одночастичному оператору, заменяя оператор числа частиц для основного состояния на $c$-число. После этого можно провести замену вида

$$
a_{0}^{\dagger} a_{0}=N-a_{+}^{\dagger} a_{+}-a_{-}^{\dagger} a_{-},
$$

используя среднее число частиц в основном состоянии. При уничтожении двух частиц в возбужденном состоянии оно равно $N_{0}+2$, а среднее значение после рождения двух частиц в возбужденном состоянии равно $N_{0}-2$. Получающийся одночастичный оператор

$$
h=a_{+}^{\dagger} a_{+}+a_{-}^{\dagger} a_{-}+g\left[N_{0}\left(a_{+}^{\dagger} a_{+}+a_{-}^{\dagger} a_{-}\right)+\left(N_{0}+3\right) a_{+} a_{-}+\left(N_{0}-2\right) a_{+}^{\dagger} a_{-}^{\dagger}\right]
$$

имеет коэффициенты на внедиагональных элементах, которые значительно отличаются от аналогичных коэффициентов боголюбовского оператора. Матричные элементы этого одночастичного оператора (17) на пространстве $|n\rangle$ равны

$$
\begin{aligned}
\langle n|h| n\rangle & =2 n+2 g N_{0} n, \\
\langle n-1|h| n\rangle & =g n\left(N_{0}+3\right), \\
\langle n+1|h| n\rangle & =g(n+1)\left(N_{0}-2\right) .
\end{aligned}
$$

Оператор $h(17)$, как показано в следующем разделе, значительно превосходит боголюбовский оператор по точности аппроксимации в широком диапазоне параметров.

\section{7. ЧИСЛЕННЫЕ РЕЗУЛЬТАТЫ}

Одночастичный боголюбовский оператор

$$
h_{\mathrm{B}}=a_{+}^{\dagger} a_{+}+a_{-}^{\dagger} a_{-}+g N_{0}\left[a_{+}^{\dagger} a_{+}+a_{-}^{\dagger} a_{-}+a_{+} a_{-}+a_{+}^{\dagger} a_{-}^{\dagger}\right]
$$

и новый одночастичный оператор $h$ такой, что

$$
h=a_{+}^{\dagger} a_{+}+a_{-}^{\dagger} a_{-}+g\left[N_{0}\left(a_{+}^{\dagger} a_{+}+a_{-}^{\dagger} a_{-}\right)+\left(N_{0}+3\right) a_{+} a_{-}+\left(N_{0}-2\right) a_{+}^{\dagger} a_{-}^{\dagger}\right],
$$

можно диагонализовать для систем $N$ частиц и любой силы взаимодействия $g$. Очевидно, разница между получающимися аппроксимациями энергии основного состояния и их отклонения от точного значения становятся пренебрежимо малы при силе взаимодействия, стремящейся к нулю. Можно ожидать большего различия между

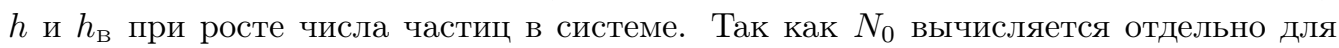




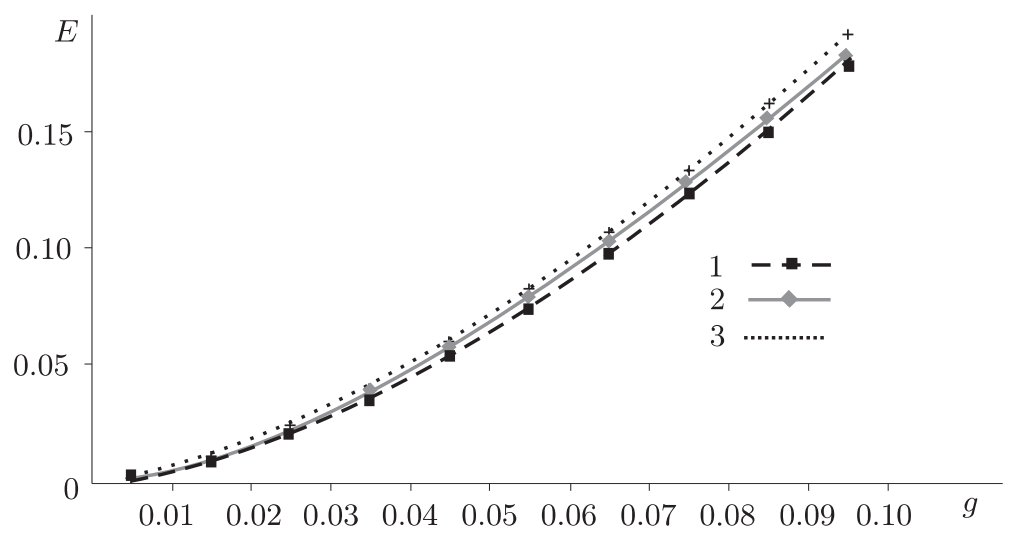

Рис. 1. Боголюбовская и новая аппроксимации энергии основного состояния для экстремально слабых сил взаимодействия. Так как в этой области доминирует кинетическая энергия, согласие с точной энергией основного состояния для обоих случаев не удивительно.

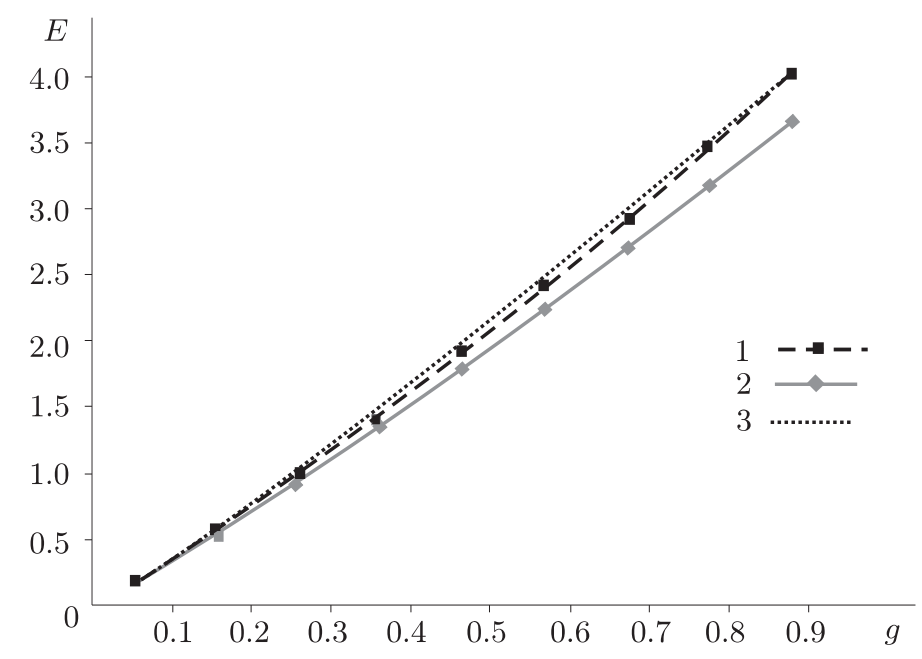

Рис. 2. Боголюбовская и новая аппроксимации энергии основного состояния для сил взаимодействия, сравнимых с кинетической энергией. Результаты новой аппроксимации существенно ближе к точным, чем результаты боголюбовской аппрокимации.

каждого оператора, результаты использования двух операторов отличаются сильнее при бо́льшем числе частиц.

Результаты для $N=8$ показаны на рис. 1-3 с тремя различными по порядку величинами $g$. Для наименьшего рассмотренного значения $g, g<0.1$, результаты боголюбовской аппроксимации немного ближе к точным значениям, чем результаты новой аппроксимации. Обе аппроксимации можно считать достаточно точными. Имеется очень малое истощение состояния с нулевым импульсом, при котором $N_{0}$ 


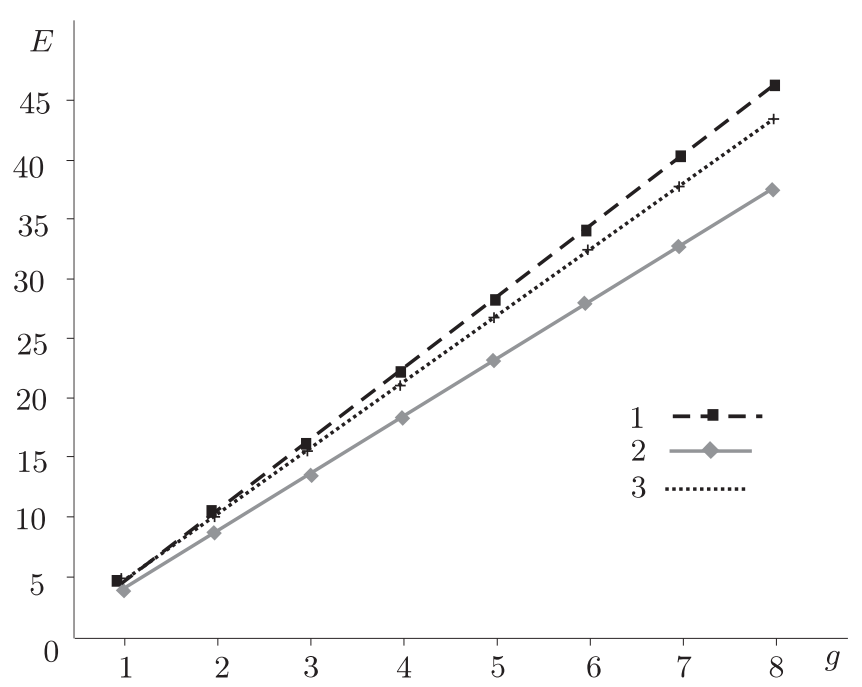

Рис. 3. Боголюбовская и новая аппроксимации энергии основного состояния для больших сил взаимодействий. Как и ожидалось, результаты аппроксимации становятся менее точными с ростом сил взаимодействия. Новые результаты все еще ближе к точным значениям, чем результаты боголюбовской аппроксимации.

имеет минимальное значение, равное 7.9 для данного диапазона $g$. Это отвечает истощению состояния с нулевым импульсом от $0.1 \%$ до $1 \%$ для $g$ из данной области.

Для больших значений $g$ (от 0.1 до 1) ошибка при боголюбовской аппроксимаци вырастает до 10\%, тогда как отклонение новых результатов от точных значений ограничено 1\%. В этой области истощение состояния с нулевым импульсом достигает $10 \%$ для боголюбовского оператора и лишь $5 \%$ для $h(17)$, как показано на рис. 4 . Для еще бо́льших значений $g$ (от 1 до 8) отклонение результатов новой аппроксимации от точных значений лежит в рамках от $2 \%$ до $6 \%$, тогда как для боголюбовской от $10 \%$ до $18 \%$. Истощение уровня с нулевым импульсом насыщается при $N=8$

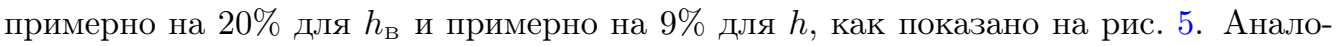
гично, при $N=16$ боголюбовские результаты немного ближе к точным значениям, чем результаты для $h$ при малых энергиях $g$, но ошибка боголюбовской аппроксимации возрастает до $13.9 \%$ при $g=8$, тогда как ошибка для $h$ составляет всего $1.4 \%$. При $g=8$ величины соответствующего истощения для для $h$ и $h_{\text {в }}$ составляют $12.5 \%$ и $18 \%$ соответственно.

Результаты для $N=32$ показаны на рис. 6 и 7 . Новые результаты опять в основном ближе к точным значениям, чем при боголюбовской аппроксимации. Разница между боголюбовским приближением и точным значением растет от $6 \%$ до $12 \%$ при возрастании $g$ от 1 до 8, в то время как для новой аппроксимации она убывает от $2 \%$ до $0.2 \%$ в том же диапазоне сил взаимодействия.

Здесь, однако, истощения состояния с нулевым импульсом практически одинаковы $-17.9 \%$ для $h_{\text {в } п р и ~} g=8$ и $17.1 \%$ для $h_{1}$. Для $N=64$ аналогичные результаты 


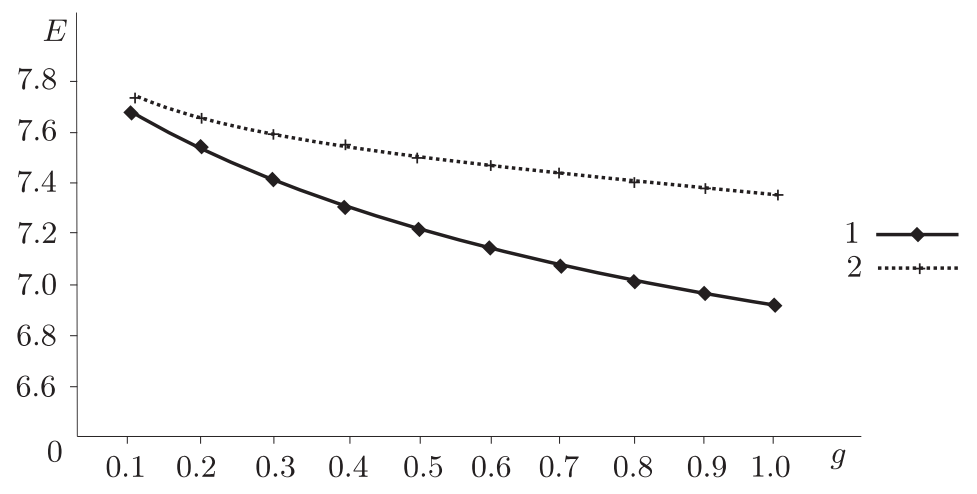

Рис. 4. Истощение основного состояния при двух аппроксимациях для сил взаимодействия, ме́ньших кинетической энергии. Среднее число частиц в основном состоянии найдено с применением обоих методов при выполнении требования совпадения общего числа частиц с желаемым числом частиц. При всех рассмотренных параметрах истощение слабее при новой аппроксимации.

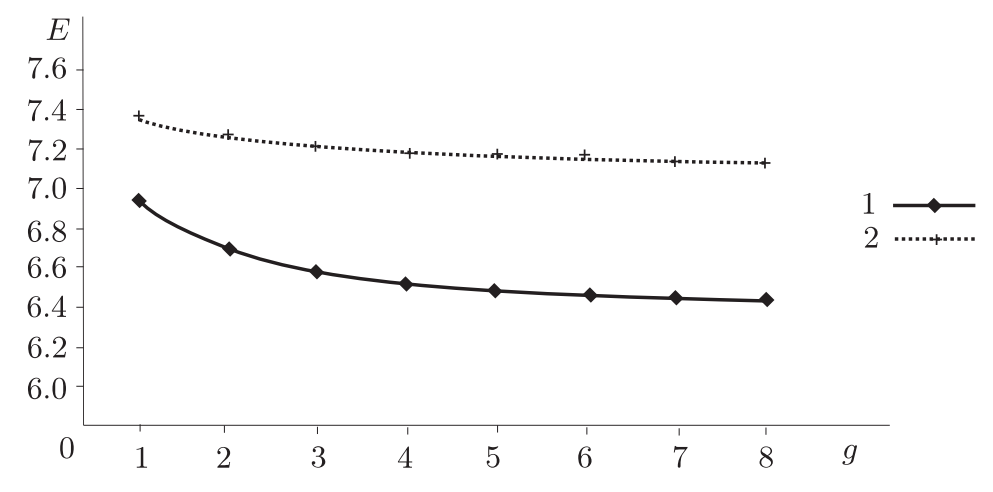

Рис. 5. Истощение основного состояния при двух аппроксимациях для сил взаимодействия, бо́льших кинетической энергии. Среднее число в основном состоянии найдено для обоих методов при помощи требования совпадения общего числа частиц с желаемым числом частиц. Видна неожиданная насыщенность истощения для обоих методов.

получены для энергий, указанных на рис. 8-10. Для наименьших значений $g$ боголюбовские результаты снова несколько ближе к точным, чем новые. Для более сильных взаимодействий с $g$ от 0.1 до 1 новые результаты отличаются от точных менее чем на $1 \%$, тогда как боголюбовские отклоняются до 4\%. Для наибольших значений $g$ соответствующие ошибки равны $7.6 \%$ для новых результатов и $11.6 \%$ для боголюбовских. Однако при данном значении $N$ истощение состояния с нулевым импульсом становится для $h$ бо́льшим, чем для $h_{\mathrm{B}}$, с насыщенностью $16.6 \%$ для боголюбовской и $26 \%$ для новой аппроксимации. Следовательно, является ли новая аппроксимация более близкой к точным результатам, чем боголюбовская, определяется не степенью истощения, а скорее силой взаимодействия. 


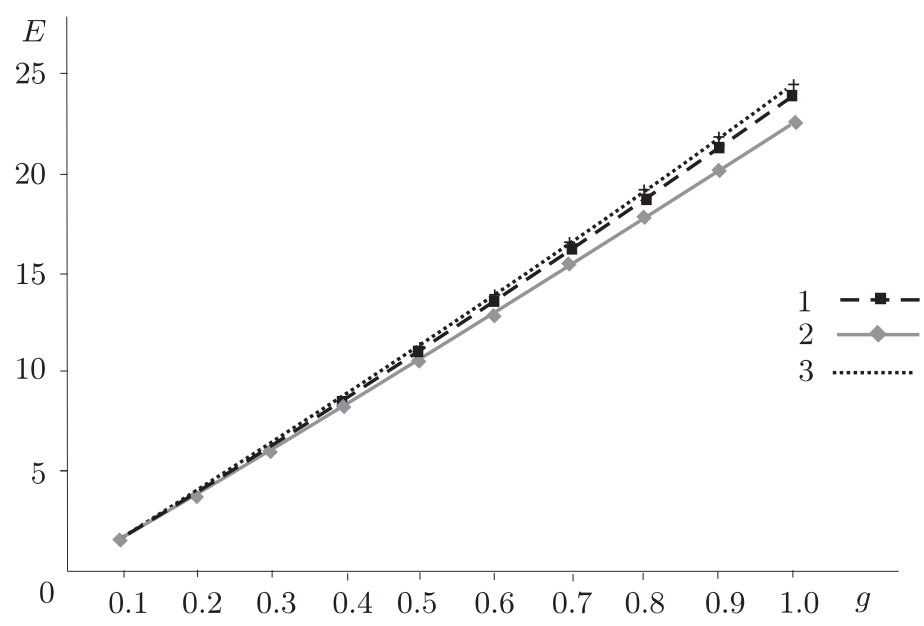

Рис. 6. Боголюбовская и новая аппроксимации энергии основного состояния для сил взаимодействия, сравнимых с кинетической энергией. Результаты новой аппроксимации значительно точнее, чем боголюбовской. Хотя в этой области новые результаты существенно ближе к точным, чем боголюбовские, степень их согласия с точными исключительна для данного числа частиц.

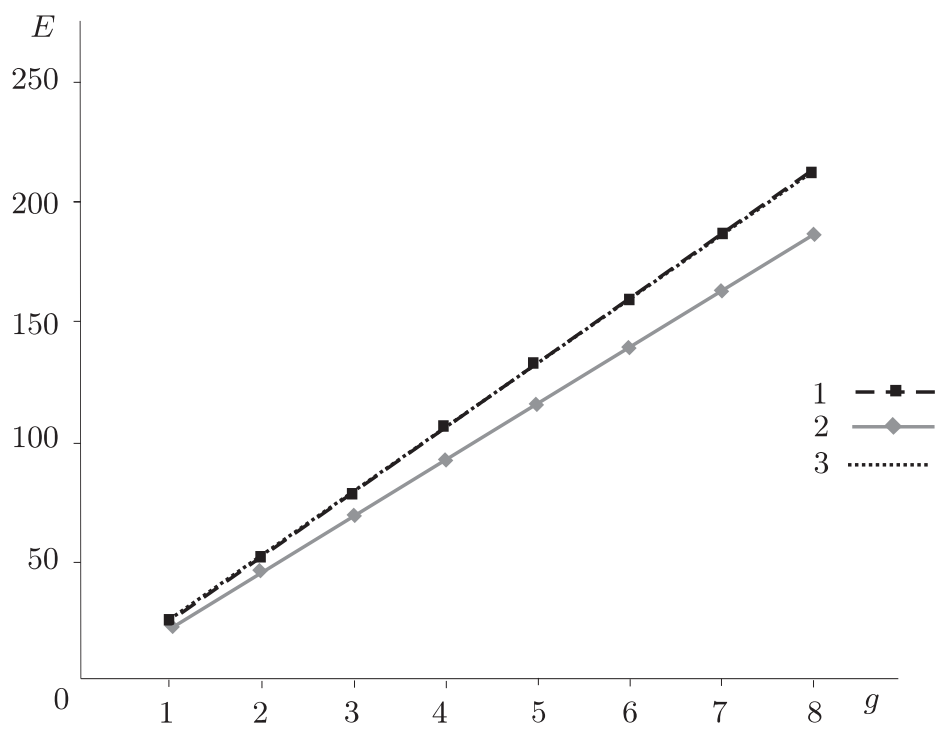

Рис. 7. Боголюбовская и новая аппроксимации энергии основного состояния для больших сил взаимодействия. Как и ожидалось, результаты аппроксимации становятся менее точными с ростом сил взаимодействия. Новые результаты остаются более точными, чем при боголюбовской аппроксимации. 


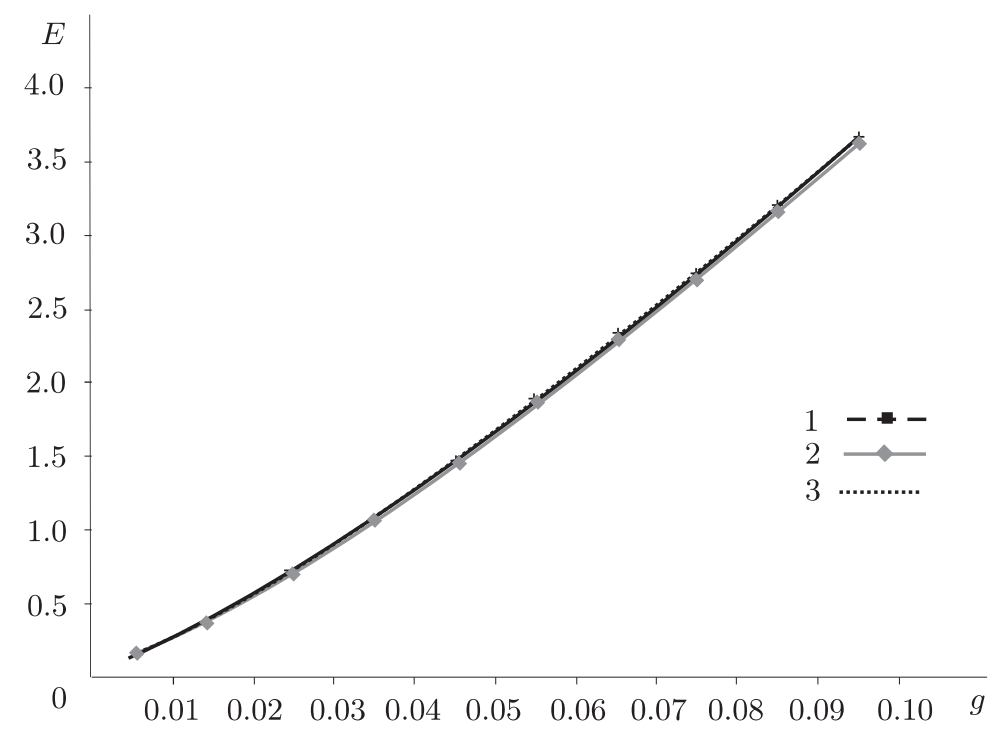

Рис. 8. Боголюбовская и новая аппроксимации энергии основного состояния для экстремально слабых сил взаимодействия. Так как в этой области доминирует кинетическая энергия, согласие с точной энергией основного состояния для обоих случаев не удивительно.

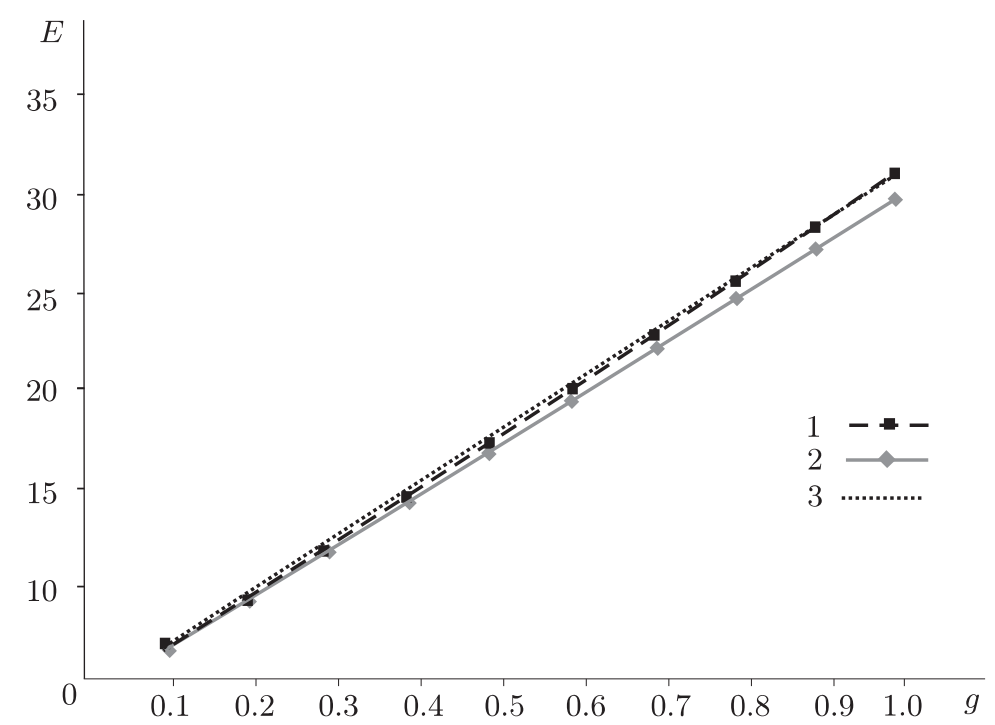

Рис. 9. Боголюбовская и новая аппроксимации энергии основного состояния для сил взаимодействия, сравнимых с кинетической энергией. Результаты новой аппроксимации существенно ближе к точным, чем результаты боголюбовской аппрокимации. 


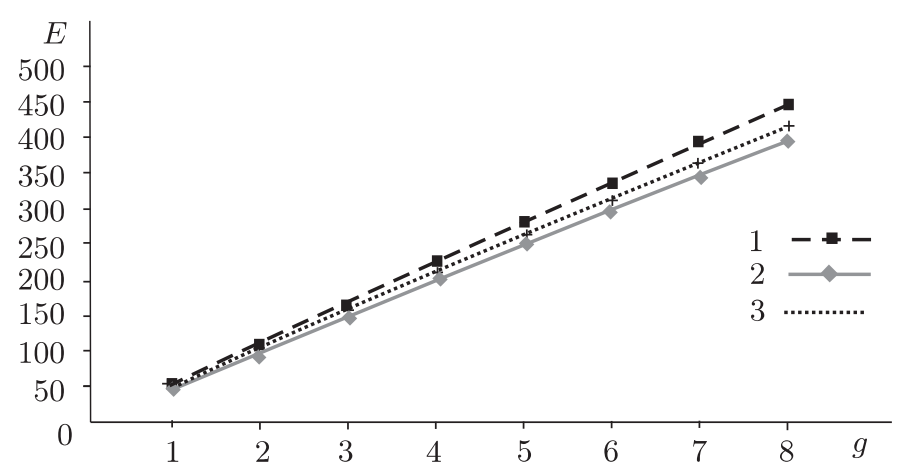

Рис. 10. Боголюбовская и новая аппроксимации энергии основного состояния для больших сил взаимодействий. Как и ожидалось, результаты аппроксимации становятся менее точными с ростом сил взаимодействия. Новые результаты все еще ближе к точным значениям, чем результаты боголюбовской аппроксимации.

\section{8. ИТОГИ}

Ранее боголюбовская аппроксимация была применена для простой одномерной бозонной модели. Преимущество этой аппроксимации состоит в замене двухчастичного взаимодействия на одночастичный оператор. Расхождения, связанные с заменой операторов рождения и уничтожения в состоянии с нулевым импульсом, как оказалось, увеличиваются с ростом сил взаимодействия. Аналогичный боголюбовскому одночастичный оператор был получен с помощью процедуры с участием только оператора числа частиц для состояния с нулевым импульсом. Результаты использования нового оператора систематически оказываются более точными, чем при использовании боголюбовского оператора, исключение составляет лишь случай крайне слабых сил взаимодействия. Построенная процедура может оказаться применима и к более реалистичным моделям, в которых точные вычисления невыполнимы.

\section{Список литературы}

[1] K. B. Davis, M.-O. Mewes, M.R. Andrews, N. J. van Druten, D. S. Durfee, D. M. Kurn, W. Ketterle, "Bose-Einstein condensation in a gas of sodium atoms", Phys. Rev. Lett., 75:22 (1995), 3969-3973.

[2] V. A. Zagrebnov, J. B. Bru, "The Bogoliubov model of weakly imperfect Bose gas", Phys. Rep., 350:5-6 (2001), 291-434.

[3] E. H. Lieb, R. Seiringer, J. P. Solovej, J. Yngvason, The Mathematics of the Bose Gas and Its Condensation, Oberwolfach Seminars, 34, Birkhäuser, Basel, 2005.

[4] A. F. Verbeure, Many Body Boson Systems. Half a Century Later, Theoretical and Mathematical Physics, 2011, Springer, London, 2011.

[5] Н. Н. Боголюбов, "К теории сверхтекучести", Изв. АН СССР. Сер. физ., 11:1 (1947), $77-90$. 
[6] A. Rovenchak, "Weakly-interacting bosons in a trap within approximate second quantization approach", J. Low Temp. Phys., 148:3 (2007), 411-416.

[7] W. H. Bassichis, L. L. Foldy, "Analysis of the Bogoliubov method applied to a simple boson model", Phys. Rev., 133:4A (1964), A935-A943.

[8] M. Razavy, R. B. Ludwig, "Clustering of levels in a solvable many-body problem with a degenerate ground state", Phys. Rev. A, 33:3 (1986), 1519-1524.

[9] E. Krotscheck, M. D. Miller, J. Wojdylo, "Variational approach to the many-boson problem in one dimension", Phys. Rev. B, 60:18 (1999), 13028-13037.

Поступила в редакцию 23.10.2015, после доработки 19.04.2016 